\title{
La politique sociale en Allemagne 1945-1994
}

Geschichte der Sozialpolitik in Deutschland seit 1945

\section{Rainer Hudemann}

\section{(2) OpenEdition}

Journals

Édition électronique

URL : http://journals.openedition.org/travailemploi/4611

DOI : 10.4000/travailemploi.4611

ISSN : 1775-416X

Éditeur

DARES - Ministère du Travail

\section{Édition imprimée}

Date de publication : 30 mars 2007

ISSN : 0224-4365

\section{Référence électronique}

Rainer Hudemann, «La politique sociale en Allemagne 1945-1994 », Travail et Emploi [En ligne], 110 | Avril-Juin 2007, mis en ligne le 26 avril 2011, consulté le 22 septembre 2020. URL : http:// journals.openedition.org/travailemploi/4611 ; DOI : https://doi.org/10.4000/travailemploi.4611 
raient de l'exemple de Douki, Feldman et Rosental en explorant comment les liens internationaux et les mutations de l'environnement international changent la structure et le rôle de l'État et de son rapport au monde du travail. Par ailleurs, ce contexte international se doit d'inclure les États-Unis, (qui figurent très peu dans ce volume) et l'Empire français. Le texte de Florence Renucci sur l'application curieuse du Code du travail dans l'AOF, et le témoignage de Jean-Paul Chaze sur sa propre expérience en tant que directeur adjoint en Polynésie, suggèrent l'importance de la France d'outre-mer dans cette histoire. L'oubli le plus surprenant dans ce volume est le manque relatif de nouvelles recherches sur les ouvriers, les syndicats, et leurs rapports au ministère. Nous apprenons plus à propos du patronat qu'à propos du travail. Finalement, l'histoire du ministère et de l'intervention de l'État dans le monde du travail après les Trente Glorieuses ne vient que de commencer. Ici, l'écriture des témoins est particulièrement utile puisque la plupart de leurs observations concernent les défis qu'ils ont affrontés pendant ces trois dernières décennies de croissance lente, désindustrialisation, et haut chômage. Ce volume, donc, servira de catalyseur pour de nouvelles explorations, alors même qu'il est un dépôt extraordinairement riche d'idées, d'observations et de recherches récentes.

\section{La politique sociale en Allemagne 1945-1994 Geschichte der Sozialpolitik in Deutschland seit 1945}

Éditeur: ministère fédéral des Affaires sociales et des Archives fédérales, Bundesministerium für Arbeit et Bundesarchiv, 11 vols

Nomos, 2001-2007.

Band 1: Grundlagen der Sozialpolitik.

Band 2: 1945 - 1949 Die Zeit der Besatzungszonen. Sozialpolitik zwischen Kriegsende und der Gründung zweier deutscher Staaten, hg. v. Prof. Dr. Udo Wengst, 2001.

Band 3: 1949 - 1957 Bundesrepublik Deutschland, Bewältigung der Kriegsfolgen, Rückkehr zur sozialpolitischen Normalität, hg. v. Prof. Dr. Günther Schulz, 2004.

Band 4: 1957 - 1966 Bundesrepublik Deutschland. Sozialpolitik im Zeichen des erreichten Wohlstands, hg.v. Prof. Dr. Michael Ruck, PD Dr. Marcel Boldorf, à paraître.

Band 5: 1966 - 1974 Bundesrepublik Deutschland, Eine Zeit vielfältigen Aufbruchs, hg. v. Prof. Dr. Hans Günter Hockerts, 2007.

Band 6: 1974 - 1982 Bundesrepublik
Deutschland. Neue Herausforderungen, wachsende Unsicherheit, hg. v. Prof. Dr. Martin H. Geyer, 2007.

Band 7: 1982 - 1989 Bundesrepublik Deutschland, Finanzielle Konsolidierung und institutionelle Reform, hg. v. Prof. Dr. Manfred G. Schmidt, 2005.

Band 8: 1949 - 1961 Deutsche Demokratische Republik. Im Zeichen des Aufbaus des Sozialismus, Hg. im Auftrag des Instituts für Zeitgeschichte München_Berlin: Dr. Dierk Hoffmann, PD Dr. Michael Schwartz, 2004.

Band 9: 1961 - 1971 Deutsche Demokratische Republik. Politische Stabilisierung und wirtschaftliche Mobilisierung, hg. v. Prof. Dr. Christoph Kleßmann, 2006.

Band 10: 1971 - 1989 Deutsche Demokratische Republik. Bewegung in der Sozialpolitik, Erstarrung und Niedergang, hg. v. PD Dr. Christoph Boyer, Prof. Dr. Klaus_Dietmar Henke z, Dr. Peter Skyba, 2007.

Band 11: 1989 - 1994 Bundesrepublik Deutschland. Sozialpolitik im Zeichen der Vereinigung, hg.v. Prof. Dr. Gerhard A. Ritter, 2007.

\section{Lu par Rainer Hudemann, universität des Saarlandes}

Par rapport à la France, la situation de la politique sociale allemande en 1945 fut très différente. Ceci non seulement à la suite du partage du pays qui s'amorça d'abord rapidement dans la réalité et ensuite juridiquement. L'Allemagne revêtait en Europe une position de pionnière en politique sociale depuis les années 1880, quand l'État commença à fondre les multiples traditions en un système cohérent d'assurances sociales. Mais ce fut un système dont les structures reflétaient, dans l'organisation et dans les conditions différentes selon les couches sociales et les branches économiques, la société de l'époque de la révolution industrielle. Certains des grands débats actuels en RFA tournent toujours autour de difficultés découlant de cet héritage.

Précisément parce que la France fut plus lente à développer et à mettre en œuvre un système comparable, elle eut en 1944-1946 l'avantage de la modernité: elle avait moins de bagages de traditions et de structures ancrées à surmonter que l'Allemagne. Avec le plan de Sécurité sociale de Pierre Laroque, elle rejoignit l'état international du débat social, parti en 1942 du plan Beveridge et de sa conception d'un système unique d'assurances sociales, intégrées dans une politique générale de sécurité sociale pour toute la société. L'Allemagne fut à un point de rupture fondamental à la fin de la guerre et du régime nazi. Mais les chances qui semblaient 
en découler pour une refonte profonde du système de politique sociale furent, après la fondation de la RFA, partiellement abandonnées. La modernisation notamment des assurances sociales dût partir des bases anciennes en RFA, dans un contexte plus complexe en RDA.

L'ambitieux et impressionnant ouvrage organisé par les soins du ministère fédéral des Affaires sociales et des Archives fédérales analyse, sur environ 13000 pages, l'évolution de la politique sociale dans les deux Allemagnes, ainsi que la période d'unification 1989-1994(1). Les responsables des onze volumes sont historiens et rédigent eux-mêmes des parties considérables, la cinquantaine d'autres auteurs, éminents spécialistes de leur domaine, venant des sciences historiques, sociales, juridiques et politiques.

Dans le premier volume, les directeurs de l'ouvrage situent, ensemble avec M. Stolleis et F.X. Kaufmann, la politique sociale des deux États allemands dans leur contexte international et dans la longue durée depuis le Moyen Âge, analysent les structures administratives et développent les conceptions structurelles. Les autres volumes, dont les volumes $2-3,5$ et 7-9 sont parus jusqu'en mars 2007, suivent très largement un plan identique couvrant un grand éventail de la politique sociale. Le directeur de chaque volume situe les conditions contextuelles et les conceptions de base pour la période concernée. Chaque auteur est responsable de son domaine pour plusieurs volumes, ce qui confère une grande cohérence à tout l'ouvrage. Toutefois, selon l'importance d'une période clef pour un domaine particulier, ce dernier y est parfois présenté dans sa longue durée. Cette grille d'un plan chronologique général de l'ouvrage et une orientation systématique permettent de faire ressortir aussi bien les structures séculaires que la dynamique de leur évolution. L'approche est institutionnelle et étatique, les acteurs non-étatiques apparaissant donc principalement dans leur rôle par rapport à l'action de l'État.

Sont ainsi systématiquement analysés (ne peuvent être cités qu'une partie des auteurs, dont les autres publications seront également fort utiles au lecteur intéressé par un sujet particulier): le droit et la protection du travail et la participation dans les entreprises (R. Richardi, D. Bethge, L. Wienhold, W. Thiel, P. Hübner); le marché du travail, sa régulation pour la RDA et le problème du chômage pour la RFA (G. Schmid, N. Wiebe, F. Oschmansky, D. Hoffmann, J. Rosler); l'organisation et le droit

(1) Geschichte der Sozialpolitik in Deutschland seit 1945, éd. Bundesministerium für Arbeit et Bundesarchiv, 11 volumes, environ 1000 à $1250 \mathrm{pp}$ et $1 \mathrm{CD}$-Rom par volume, BadenBaden: Nomos 2001-2007 (dont sept parus au moment de la rédaction de cette note de lecture, les autres paraissant au cours de l'été 2007). des prestations sociales (P. Krause, H. Zacher, D. Hoffmann); la politique des prix en RDA (H. Buck, M. Landsman, A. Steiner); l'assurance vieillesse et invalidité ainsi que les systèmes pour des catégories particulières en RDA telles la Stasi (W. Schmähl, D. Hoffmann); les systèmes de santé et l'assurance-maladie (J. Wasem e. a.), l'assurance accident et les maladies professionnelles (W. Gitter e. a.), la politique pour les handicapés, l'assistance sociale (P. Trenk-Hinterberger, M. Boldorf e. a.), la politique pour la famille et la jeunesse (U. Münch, G. Helwig e. a.), la politique d'éducation - correspondant plus à la notion anglaise qu'allemande de politique sociale (O. Anweiler) -, les suites de la guerre et des dictatures, ainsi que la politique de réparation des dommages causés aux victimes du nazisme (W. Rüfner, C. Goschler, M. Schwartz); l'emploi et l'intégration des étrangers (U. Herbert, K. Hunn e. a.), les infrastructures sociales (D. Grunow, T. Olk); l'habitat (T. Harlander, G. Schulz, J. Rowell u. a.); la politique favorable au patrimoine en RFA (Y. Dietrich), la politique sociale d'entreprise en RDA (P. Hübner), enfin la politique sociale internationale (E. Eichenhofer, P. Köhler, B. v. Maydell, M. Boldorf, A. Wilczek). Chaque volume est complété par un CD-Rom, conçu pour des recherches très diversifiées et détaillées, présentant une vaste documentation - en majorité inédite - étroitement liée au texte et puisant notamment dans les archives publiques et privées ainsi que dans la presse.

L'après-guerre fut l'époque des grandes discussions sur les principes de la politique sociale. En même temps surgirent de multiples tentatives pour faire face aux suites de la guerre et des destructions, d'où pouvaient sortir des systèmes entièrement neufs comme celui pour les victimes du nazisme (2). Le Gouvernement militaire français en Allemagne entreprit dès l'hiver 1945-1946 une réforme en profondeur des assurances sociales dans sa zone d'occupation, élément clef de sa politique de démocratisation de la société allemande destinée à garantir mieux qu'auparavant la sécurité de la France à l'avenir (3). Cette convergence partielle des systèmes français et allemand resta un épisode limité à 1945-1949, bien qu'une partie des réformes françaises fût réalisée dans les deux décennies suivantes par d'autres moyens et qu'à la fin du millénaire, pratiquement oubliées, elles n'aient rien perdu de

(2) Cf. l'ouvrage principalement juridique: Die Wiedergutmachung nationalsozialistischen Unrechts durch die Bundesrepublik Deutschland, Hg. Bundesminister der Finanzen in Verbindung mit Walter Schwarz, 7 volumes, Munich, Beck, 1974-2000.

(3) R. Hudemann, Sozialpolitik im deutschen Südwesten zwischen Tradition und Neuordnung 1945-1953. Sozialversicherung und Kriegsopferversorgung im Rahmen französischer Besatzungspolitik, Mayence, v. Hase \& Kœhler, 1988. 
leur actualité structurelle dans le vaste débat public sur la réforme de l'assurance-maladie allemande.

Des problèmes immédiats en 1945 sortirent de vastes conceptions que l'ouvrage retrace aussi bien dans les contenus que dans leur cadre politique, économique et social. L'intégration difficile des 11 millions de réfugiés de 1'Est n'en fut qu'un exemple, contribuant en même temps beaucoup à la modernisation de la société allemande. En RFA, les années 1950 apportèrent l'intégration d'un nombre croissant de prestations sociales dans la dynamique générale de l'évolution du niveau de vie, dont les rentes en 1957. De même, la forte position des syndicats et de la participation devint un élément clef de la stabilisation de la démocratie ouest-allemande. La politique de soutien à l'acquisition d'un patrimoine immobilier ou d'épargne fut un exemple parmi d'autres pour consolider les bases de l'Etat social au-delà des prestations immédiates, modifiant en même temps les mentalités au moment du grand essor et des transformations qualitatives de la consommation. Vint l'époque du nivellement des différences entre ouvriers et salariés à partir des années 1960 et du passage de la politique sociale à l'ambition d'une «politique de société», symbolisée par la culmination de la part du budget social à un tiers du BIP vers 1975. Les volumes retracent de façon précise les mesures concrètes dans lesquelles se matérialisèrent les grandes tendances du siècle, comme l'expansion de l'État interventionniste et ses limites. Les évolutions profondes que connut la position des femmes dans la société marquent la quasi-totalité des sujets traités, permettant ainsi de saisir la multitude des domaines et les divergences, les succès et les retards qui les caractérisent.

La RDA, refusant jusqu'aux années 1970 officiellement toute politique sociale dont seul un régime capitaliste aurait besoin pour «réparer» ses dégâts, développa en fait très tôt des systèmes tout à fait particuliers dans la mesure où ils étaient situés entre les vieilles traditions allemandes et les influences soviétiques. Un marché n'existait plus, ni un partenariat dans les entreprises, les syndicats faisant partie des organes staliniens de «transmission » entre le parti et les masses. D'où les énormes difficultés d'adaptation de la population est-allemande quand elle fut propulsée dans un système d'économie sociale du marché en 1990. Les évolutions et aléas de la politique sociale permettent de saisir la complexité des bases du régime, manquant de légitimité par des élections et étant obligé de se consolider par d'autres moyens. Ceci amena la revalorisation officielle de la politique sociale à partir des années 1970, bien qu'elle fût toujours subordonnée à la politique économique, reléguant par exemple la protection contre les accidents au second plan. La RDA continuait également à exclure des groupes traditionnellement mis en marge de la société comme les jeunes déviants ou les prostituées. La position ambivalente des femmes entre une obligation de travailler vu les bas salaires et une position très traditionnelle à la maison fit partie des nombreuses contradictions inhérentes à cette société. Le maintien d'un niveau néanmoins important du secteur social alors que la productivité ne permettait plus de le financer, contribua pour beaucoup au déclin de ce régime.

La politique sociale au niveau européen prend, au cours de l'analyse, la place croissante qui lui revient aux niveaux nationaux. Le volume sur l'époque de l'unification (à paraître en été 2007) approfondira cette perspective tout en apportant un volet fondamental à l'explication des différences actuelles entre les deux parties de la RFA.

De par son approche méthodique, le livre ne cherche pas à couvrir tous les domaines sociaux dans le sens de Beveridge, l'urbanisme étant par exemple abordé surtout dans le cadre de l'habitat. La comparaison internationale sur le plan de 1'histoire sociale aussi bien que politique est souvent approfondie; pour d' autres sujets, le lecteur trouvera des informations précieuses pour pousser lui-même plus loin la réflexion. Ainsi, le rôle très différent de la participation dans la culture d'entreprise en Allemagne permet de comprendre des divergences franco-allemandes profondes et séculaires, touchant tous les domaines de la société. Mais l'ambition de l'ouvrage est très vaste, et le résultat est à la hauteur de cette ambition: précis, toujours analytique, innovateur dans la réflexion et bien situé dans le contexte international - aussi bien pris dans le sens de la comparaison que dans celui du niveau international de politique sociale. Le lecteur y trouve une mine richissime d'informations pour tous les domaines d'histoire sociale. L'enrichissement intellectuel et scientifique pour la réflexion aussi bien historique que systématique sur les grands sujets contemporains de la politique sociale mérite de dépasser les limites artificielles de la langue allemande. 